\title{
The Response of Chick Sensory Neurons to Brain-Derived Neurotrophic Factor
}

\author{
Alun M. Davies, H. Thoenen, and Yves-Alain Barde \\ Department of Anatomy, St. George's Hospital Medical School, London, and Department of Neurochemistry, \\ Max-Planck-Institute for Psychiatry, Munich, West Germany
}

To determine the spectrum of activity of brain-derived neurotrophic factor (BDNF) among first-order sensory neurons, explants of the nine distinct populations of sensory neurons from embryonic chicks of 3-14 d incubation (E3-E14) were grown in collagen gels with and without BDNF in the culture medium. The explants responded to BDNF with profuse neurite outgrowth and comprised those in which neurons are derived from neural crest (the dorsomedial part of the trigeminal ganglion, rostromedial part of the trigeminal mesencephalic nucleus, jugular ganglion, and lumbosacral dorsal root ganglia) and from epibranchial placodes (the ventrolateral part of the trigeminal ganglion and the geniculate, petrosal, and nodose ganglia). This response was first clearly observed in the ventrolateral trigeminal and nodose ganglia as early as $\mathbf{E 4}$, but did not appear until later in other explants. The dorsomedial trigeminal, jugular, and geniculate ganglia were the latest to develop a response, which was not apparent until E8. In all explants the response was maximal between $\mathrm{E} 10$ and $\mathrm{E12}$, and there was a decline in the magnitude of the response from E12 to E14. Although explants of the vestibular ganglion failed to respond to BDNF, the survival and growth of vestibular neurons in dissociated neuron-enriched cultures were promoted by BDNF. To investigate whether all first-order sensory neurons respond to BDNF or whether BDNF-responsive neurons comprise a distinct subset, we studied the influence of BDNF and NGF on the survival and growth of the placode-derived and the neural crest-derived neurons of the trigeminal ganglion in dissociated neuron-enriched culture. Whereas the great majority of ventrolateral trigeminal neurons (placode-derived) survived and grew in response to BDNF, only about $7 \%$ of dorsomedial trigeminal neurons (neural crest-derived) survived with BDNF. These findings demonstrate that although BDNF-responsive neurons are present in all populations of first-order sensory neurons, the proportion of responding neurons in these populations varies.

Sensory neurons connect specific types of sensory receptors in the periphery with particular groups of second-order neurons in the CNS. During the establishment of these connections, from 30 to $70 \%$ of the neurons originally present die (Oppenheim, 1981), and this loss is believed to match the number of neurons to the requirements of their target fields and to eliminate inappropriately connected neurons (Cowan et al., 1984). Considerable evidence, largely from work on NGF, suggests that neuronal survival during this critical period of development depends on the supply of a trophic factor conveyed from the target field

\footnotetext{
Received June 24, 1985; revised Nov. 14, 1985; accepted Dec. 18, 1985.

We thank Frau Cáp for technical assistance.

Correspondence should be addressed to Alun M. Davies, Department of Anatomy, St. George's Hospital Medical School, Cranmer Terrace, London SW17 ORE, England.

Copyright $($ C 1986 Society for Neuroscience $0270-6474 / 86 / 071897-08 \$ 02.00 / 0$
}

to the perikaryon by axonal transport (Berg, 1982; Thoenen and Barde, 1980).

The presence of NGF in developing cutaneous fields (A. M. Davies, $S$. Korsching, and $H$. Thoenen, unpublished observations) and in the peripheral, but not the central, branches of dorsal root ganglia (Korsching and Thoenen, 1985), together with the demonstration of the retrograde transport of endogenous NGF from the periphery (Korsching and Thoenen, 1983), suggests that NGF provides trophic support to developing sensory neurons via their peripheral processes. NGF does not, however, support the survival and growth of all types of sensory neurons: For example, the placode-derived neurons of the ventrolateral trigeminal (Davies and Lumsden, 1983; Ebendal and Hedlund, 1975), the nodose (Lindsay et al., 1985), geniculate, vestibular, and petrosal ganglia (Davies and Lindsay, 1985), and the neural crest-derived neurons of the trigeminal mesencephalic nucleus (A. M. Davies, A. G. S. Lumsden, H. Thoenen, and $\mathrm{H}$. Rohrer, unpublished observations) do not respond. Although these sensory neurons are unresponsive to NGF, there is evidence that they are supported by other neurotrophic factors present in their peripheral target fields. The enteroceptive neurons of the nodose ganglion are supported by a factor present in liver (Lindsay and Tarbit, 1979), and the proprioceptive neurons of the trigeminal mesencephalic nucleus are supported by a factor present in skeletal muscle (Davies, in press). Furthermore, these as yet unpurified factors have a negligible neuritepromoting effect on explants of the NGF-responsive cutaneous sensory neurons of the trigeminal ganglion (Davies, in press; Davies and Lindsay, 1984). Taken together, the above studies suggest that each kind of sensory neuron is supported by one of a number of neurotrophic factors present in the periphery.

Recent experiments (Yip and Johnson, 1984) showing that central rhizotomy of dorsal root ganglia (DRG) in newborn rats results in the death of a proportion of the neurons suggest that sensory neurons also require trophic support from the CNS. Little is known, however, of the molecular basis of such support. Since sensory neurons project to a variety of different kinds of second-order neurons in the CNS, it is possible that, as in the periphery, a number of different neurotrophic factors are involved. One way to begin to investigate this possibility would be to determine the spectrum of activity of a CNS-derived neurotrophic factor for sensory neurons. Brain-derived neurotrophic factor (BDNF), which has been purified from pig brain (Barde et al., 1982), appears to be such a factor. It has been shown to support the survival and growth of nodose and dorsal root ganglion neurons in culture but is inactive on sympathetic and parasympathetic neurons (Lindsay et al., 1985).

\section{Materials and Methods}

\section{Dissection}

Electrolytically sharpened tungsten needles were used to dissect lumbosacral DRG and the cranial sensory ganglia (Davies and Lindsay, 

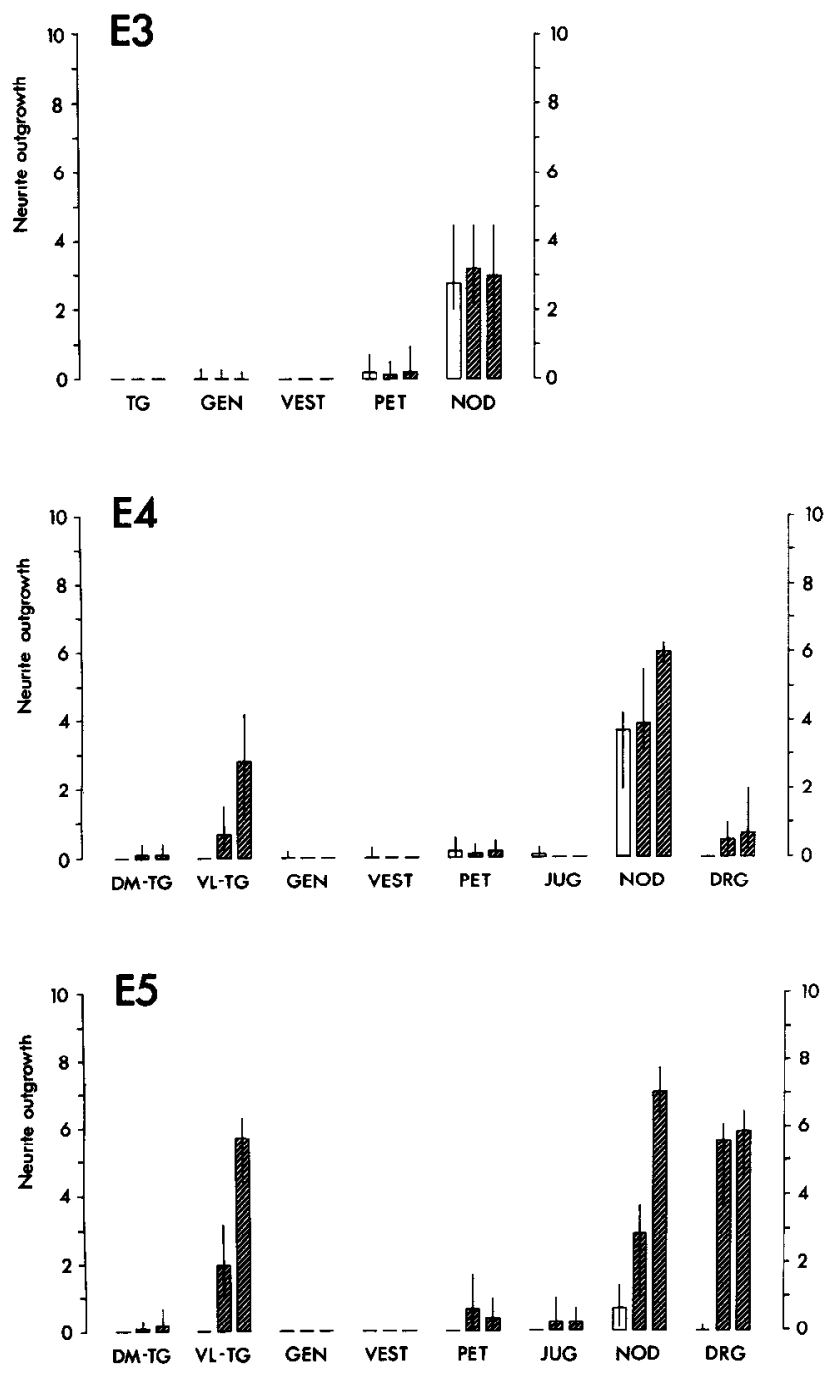

Figure 1. Bar charts showing the magnitude of neurite outgrowth from ganglion explants of E3-E14 chick embryos. Bar height, Median of neurite outgrowth scores after $24 \mathrm{hr}$ incubation; upper and lower limits of the vertical line transecting each bar, the positive and negative interquartile deviations, respectively. Three bars are drawn for each kind of explant: the first (blank bar) summarizes neurite outgrowth in control cultures; the second and third bars (hatched) summarize neurite outgrowth from explants grown in the presence of BDNF at 25 and 100 $\mathrm{ng} / \mathrm{ml}$, respectively. $T G$, Whole trigeminal ganglion at $\mathrm{E} 3$ only; $D M$ $T G$, dorsomedial pole of the maxillomandibular pole of the trigeminal ganglion; $V L-T G$, ventrolateral pole of the maxillomandibular lobe of the trigeminal ganglion; $M E S$, rostromedian part of the trigeminal mesencephalic nucleus; $G E N$, geniculate ganglion; $V E S T$, vestibular component of the vestibuloacoustic ganglion; $P E T$, petrosal ganglion; $J U G$, jugular ganglion; $N O D$, nodose ganglion; $D R G$, dorsal root ganglion.
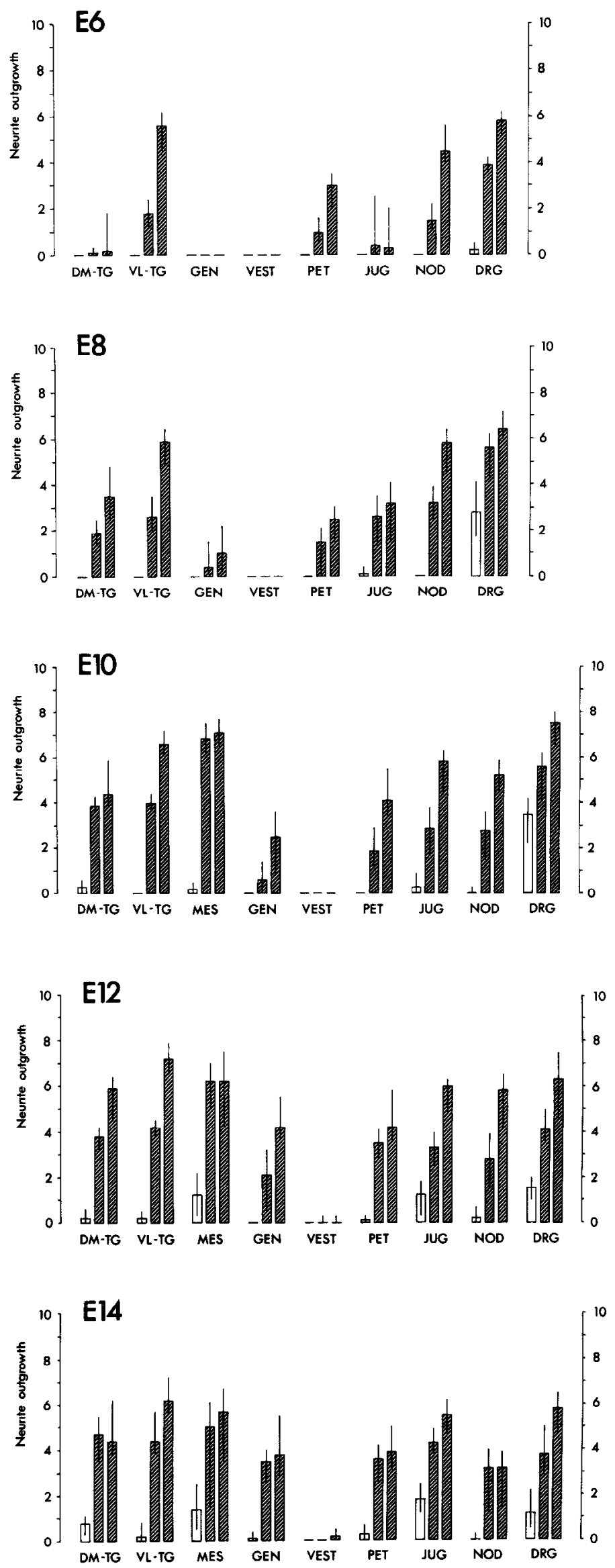


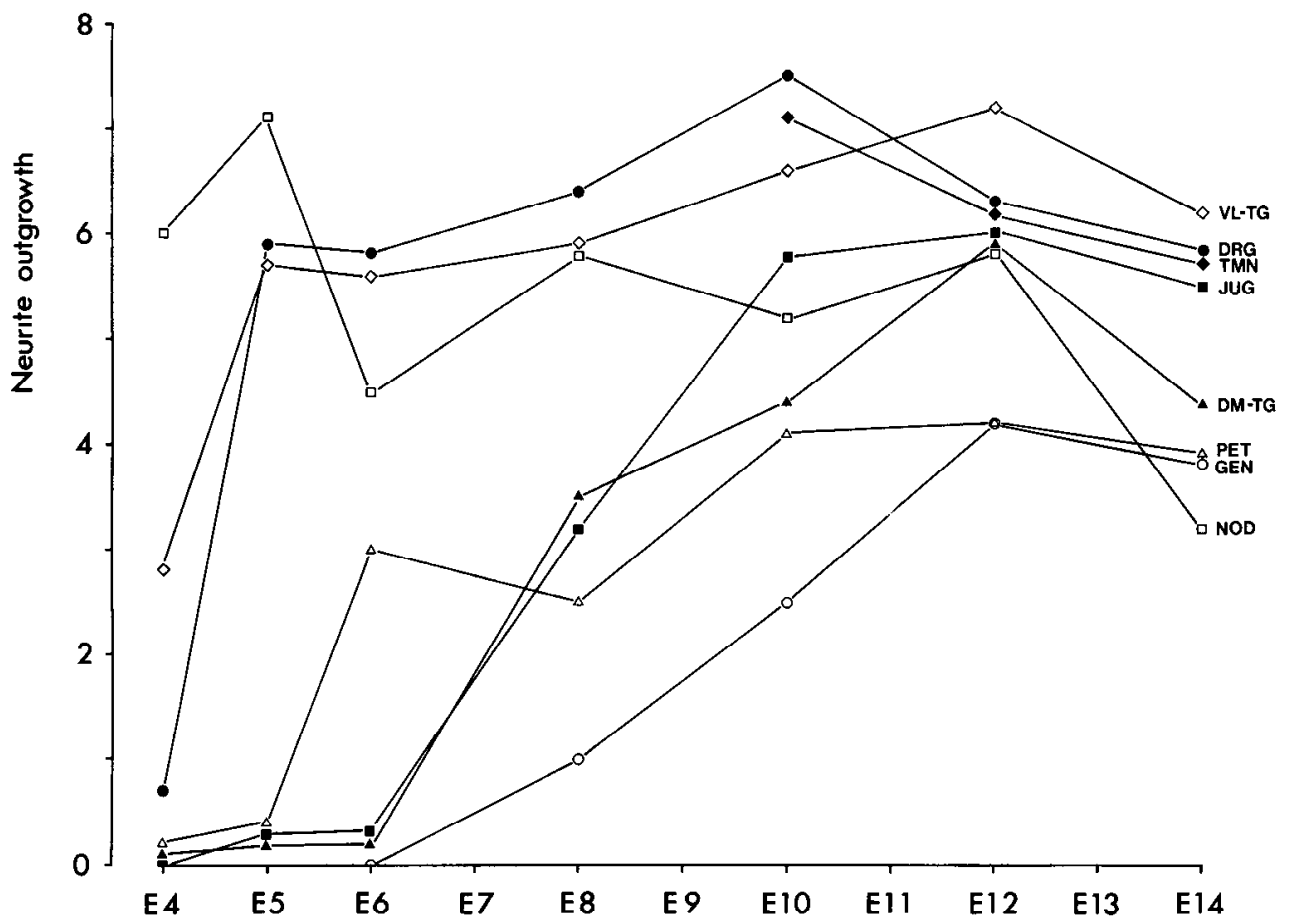

Figure 2. Graph summarizing the age-related trends in BDNF-promoted neurite outgrowth from neural crest-derived explants (solid symbols) and placode-derived explants (open symbols). The median of the neurite outgrowth scores after $24 \mathrm{hr}$ in the presence of BDNF $(100 \mathrm{ng} / \mathrm{ml})$ is plotted. Solid triangles, Dorsomedial part of the trigeminal ganglion; solid diamonds, rostromedial part of the trigeminal mesencephalic nucleus; solid squares, jugular ganglion; solid circles, DRG; open diamonds, ventrolateral part of the trigeminal ganglion; open circles, geniculate ganglion; open triangles, petrosal ganglion; open squares, nodose ganglion.
1985) from chick embryos of $4,5,6,8,10,12$, and $14 \mathrm{~d}$ incubation (E4-E14). The maxillomandibular lobe of the trigeminal ganglion was further dissected into placode-derived and neural crest-derived components, the ventrolateral pole (VL-TG), and dorsomedial pole (DMTG), respectively (Davies and Lumsden, 1983). In addition, at E3, the ganglia of placodal origin (the entire trigeminal ganglion at this stage, the geniculate, vestibular, petrosal, and nodose ganglia) were sufficiently well formed to permit dissection. The rostromedian part of the trigeminal mesencephalic nucleus (Davies, in press) was dissected from E10, E12, and E14 embryos. Paravertebral sympathetic and ciliary ganglia were dissected from E10 and E12 embryos.

\section{Explant culture}

Five explants of each type were embedded in $250 \mu$ l of collagen gel (Lumsden and Davies, 1983) in 16-mm-diameter plastic wells (Nunc multiwell dishes) and overlaid with $60 \mu$ l of heat-inactivated horse serum (Gibco) and $290 \mu \mathrm{l}$ of Fl4 medium (Barde et al., 1980). Control cultures and cultures supplemented with BDNF at concentrations of 25 and $100 \mathrm{ng} / \mathrm{ml}$ (unless otherwise stated) were set up at each age.

After $24 \mathrm{hr}$ incubation at $37^{\circ} \mathrm{C}$, the cultures were viewed by phasecontrast microscopy. The magnitude of neurite outgrowth from each explant was scored on an ordinal scale from 0 to 10 (Davies and Lumsden, 1984).

\section{Dissociated neuron-enriched cultures}

Vestibular ganglia and the DM-TG and VL-TG were trypsinized and dissociated as described previously (Davies and Lindsay, 1985). The dissociated cell suspension was enriched for neurons by the technique of differential sedimentation (Davies, in press). Neurons were grown in 35-mm-diameter plastic culture dishes (Nunc) at 2000-4000 neurons per dish. The substratum was prepared by sequentially coating dishes with polyornithine, $0.5 \mathrm{mg} / \mathrm{ml}$ (Sigma), and laminin, $10 \mu \mathrm{g} / \mathrm{ml}$ (BRL), by the methods of Collins (1978) and Edgar et al. (1984), respectively. Neurons were grown in $2 \mathrm{ml}$ of F14 medium containing heat-inactivated horse serum at $10 \%$. Control cultures and cultures supplemented with either BDNF at 10 and $50 \mathrm{ng} / \mathrm{ml}$ or NGF at 5 and $25 \mathrm{ng} / \mathrm{ml}$ were set up in duplicate. After a $48 \mathrm{hr}$ incubation, the number of neurons with neurites of lengths greater than five perikaryon diameters was counted. This number was expressed as the percentage of the number of cells plated.

\section{BDNF purification}

BDNF was purified from pig brain as described in Barde et al. (1982), with some modifications (Lindsay et al., 1985).

\section{$N G F$ purification}

NGF was purified from adult male mouse submandibular salivary glands according to the method of Bocchini and Angeletti (1969), with the modifications described in Suda et al. (1978).

\section{Results}

\section{Explant cultures}

The magnitude of neurite outgrowth from explants grown in the absence of BDNF was, with the exception of the nodose and dorsal root ganglia, low at all ages. There was moderate neurite outgrowth from control cultures of E3 and E4 nodose ganglia and E8-E12 DRG (Fig. 1).

At E3 the magnitude of neurite outgrowth from explanted ganglia was not influenced by the presence of BDNF (Fig. 1). By E4 a number of ganglia had become responsive: The VLTG and DRG showed a slight response, and neurite outgrowth from nodose ganglia in the presence of BDNF was significantly larger than that of controls (Mann-Whitney test, $p<0.01$ ). By E8, all explants except the vestibular showed a clear response to BDNF; the geniculate and DM-TG explants were the last to respond. Trigeminal mesencephalic explants at E10, the earliest age at which this nucleus can be cleanly dissected from the midbrain, showed a marked response to BDNF. For all responsive ganglia, BDNF-promoted neurite outgrowth reached a maximum between E10 and E12 and declined between E12 and E14. Figure 2 summarizes the age-related trends in the response of explants to BDNF. The typical appearance of VL$\mathrm{TG}$ and trigeminal mesencephalic explants grown with and without BDNF is shown in Figure 3.

Apart from a small number of neurites at E12 and E14, vestibular ganglion explants showed no response to BDNF throughout the period of development studied. To determine whether the lack of response of vestibular explants in the above series of experiments was due to the use of inappropriate concentrations of BDNF and/or a more stringent growth substratum requirement of vestibular neurites, E10 vestibular explants were incubated in the presence of BDNF at concentrations of 2,5 , $10,40,100,200,400$, and $800 \mathrm{ng} / \mathrm{ml}$ with and without laminin $(10 \mu \mathrm{g} / \mathrm{ml}$ ) incorporated into the collagen matrix. No neurite 

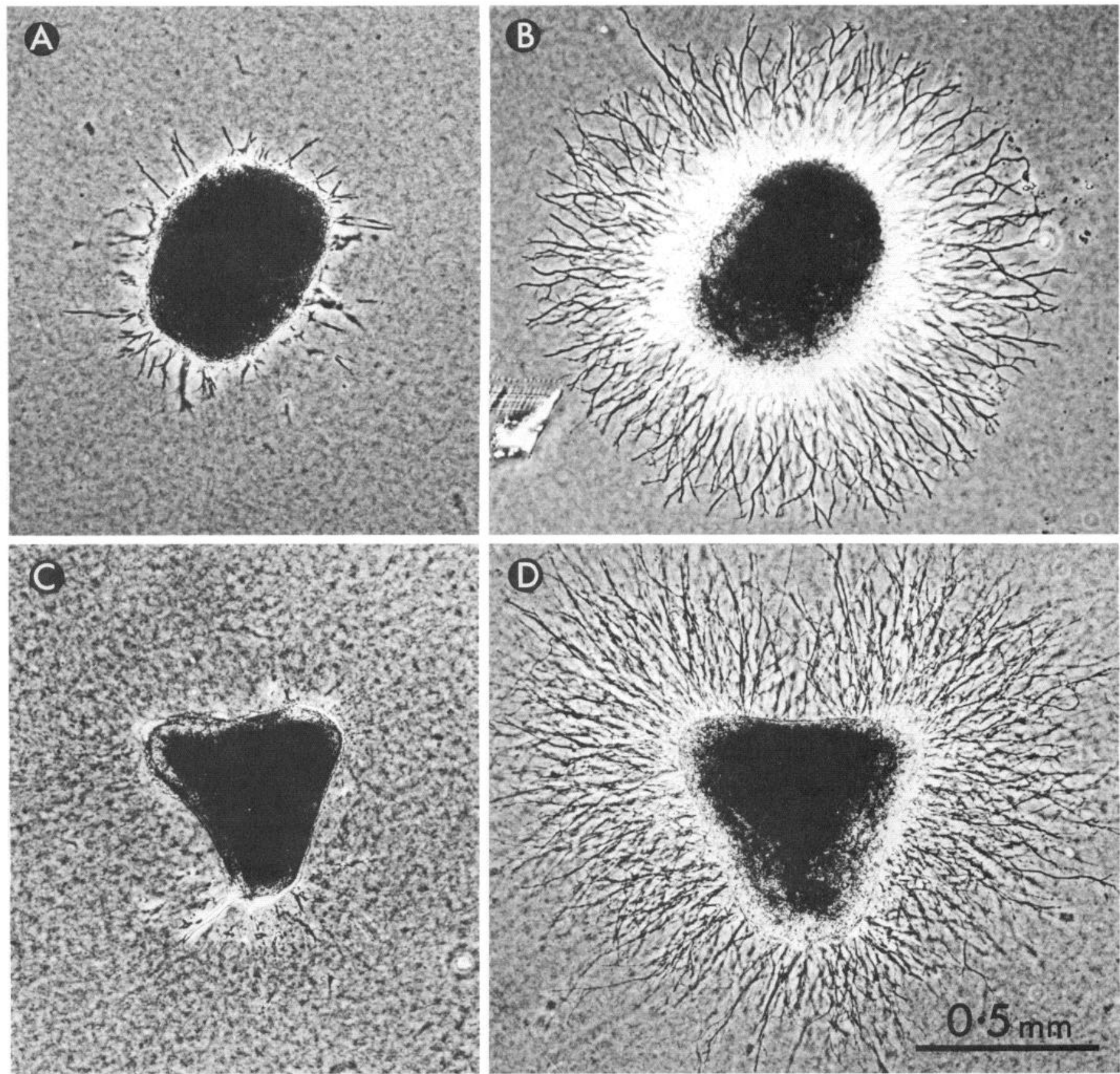

Figure 3. Phase-contrast micrographs of E10 explants of the rostromedial part of the trigeminal mesencephalic nucleus (TMN) and ventrolateral pole of the trigeminal ganglion (VL-TG) after $24 \mathrm{hr}$ in culture. $A$, VL-TG explant grown in control conditions; there is no neurite outgrowth. $B$, VL-TG explant grown in the presence of BDNF at a concentration of $100 \mathrm{ng} / \mathrm{ml}$; a dense halo of neurites is visible. $C$, TMN explant grown in control conditions; no neurites can be seen. $D$, TMN explant grown in the presence of BDNF at $100 \mathrm{ng} / \mathrm{ml}$, showing marked neurite outgrowth.

outgrowth was observed from vestibular explants under any of these conditions.

To determine the relationship between the onset of BDNF responsiveness and the onset of NGF responsiveness in populations of developing sensory neurons, we grew DRG explants of E4-E6 chick embryos with either BDNF or NGF. At E4 a slight response to BDNF was consistently observed in all experiments, but there was no response to NGF (Fig. 4). At E5 moderate neurite outgrowth was observed in response to both BDNF and NGF, and at E6 the magnitude of this response showed a further increase.

No fiber outgrowth was observed from explants of sympathetic and parasympathetic ganglia grown with BDNF.

\section{Dissociated neuron cultures}

In control cultures of E10 vestibular neurons, the majority of neurons degenerated: After $48 \mathrm{hr}$ incubation, only $1.5 \pm 0.5 \%$ (mean \pm SEM, $n=4$ ) of the neurons plated were growing. In the presence of BDNF at $50 \mathrm{ng} / \mathrm{ml}$, however, $36.5 \pm 3 \%$ of the neurons survived and grew after $48 \mathrm{hr}$ incubation. The typical appearance of these neurons is shown in Figure 5.

In control cultures of E10 VL-TG and DM-TG neurons, fewer than $1 \%$ of the neurons survived $48 \mathrm{hr}$ incubation. Whereas BDNF promoted the survival and growth of the majority of VL-TG neurons, only a small number (about 7\%) of DM-TG neurons were supported. In contrast, NGF promoted the sur- 


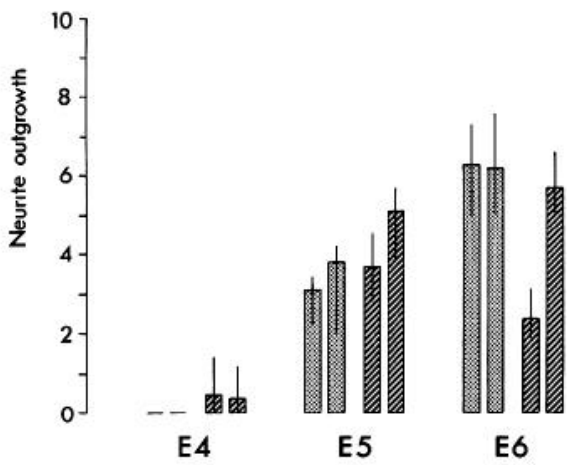

Figure 4. Bar chart showing the magnitude of neurite outgrowth from explants of E4-E6 DRG grown in the presence of either BDNF at 25 and $100 \mathrm{ng} / \mathrm{ml}$ (hatched bars) or of NGF at 1.25 and $5 \mathrm{ng} / \mathrm{ml}$ (stippled bars). Bar height, The median of neurite outgrowth scores after $24 \mathrm{hr}$ incubation; upper and lower limits of the vertical line transecting each bar, the positive and negative interquartile deviations, respectively.

vival and growth of the majority of DM-TG neurons, but only about $5 \%$ of VL-TG responded. These differences in the response of VL-TG and DM-TG neurons to BDNF and NGF were also observed at E6, E8, and E12. In accordance with the results of explant cultures, the percentage survival was greatest in E10 cultures. The quantitative E10 data are shown in Figure 6, and the typical appearance of E10 DM-TG and VL-TG neurons grown with BDNF and with NGF is shown in Figure 7. Although cell counts were usually performed after $48 \mathrm{hr}$ incubation, no significant changes were observed over the following days.

\section{Discussion}

We have shown that all populations of first-order sensory neurons contain BDNF-responsive neurons. In explant culture, BDNF elicited marked neurite outgrowth from the trigeminal mesencephalic nucleus and all sensory ganglia except the vestibular, and although unresponsive in explant culture, vestibular

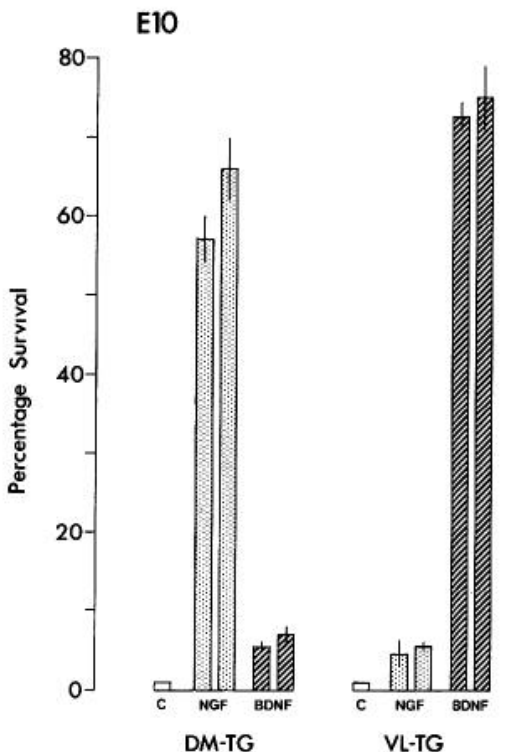

Figure 6. Bar chart showing the percentage survival of E10 dorsomedial trigeminal $(D M-T G)$ and ventrolateral trigeminal $(V L-T G)$ neurons in control cultures (blank bars), cultures supplemented with NGF at 5 and $25 \mathrm{ng} / \mathrm{ml}$ (stippled bars), and cultures supplemented with BDNF at 10 and $50 \mathrm{ng} / \mathrm{ml}$ (hatched bars) after $48 \mathrm{hr}$ incubation. Bar height, The mean, and line transecting the bars, the percentage survival in each of the duplicate cultures.

neurons were supported by BDNF in dissociated culture. The discrepancy in the action of BDNF on vestibular explants and on dissociated vestibular neurons may be due to the particularly prominent connective tissue capsule that closely invests this ganglion, hindering neurite outgrowth.

Explants differed in the age at which BDNF first elicited a response. The earliest to respond were the VL-TG, nodose, and DRG and the latest, the DM-TG, jugular and geniculate. The onset of the response, for most explants, appears to have been
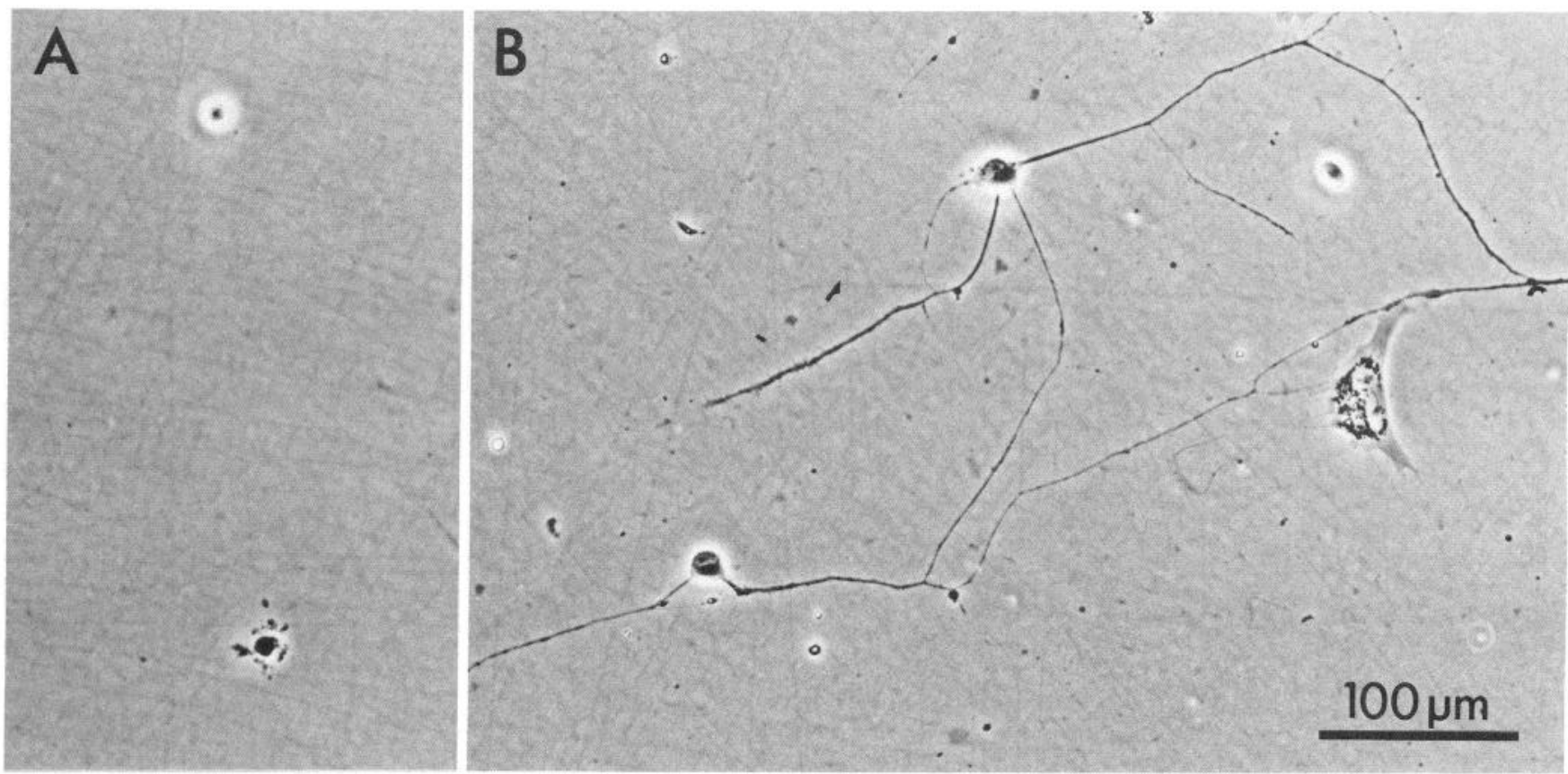

Figure 5. Phase-contrast micrographs of dissociated neuron-enriched cultures of E10 vestibular neurons after $48 \mathrm{hr}$ incubation. $A$, Control culture showing a degenerating and a phase-bright neuron without processes. $B$, Neurons growing in the presence of $\mathrm{BDNF}$ at $50 \mathrm{ng} / \mathrm{ml}$. 

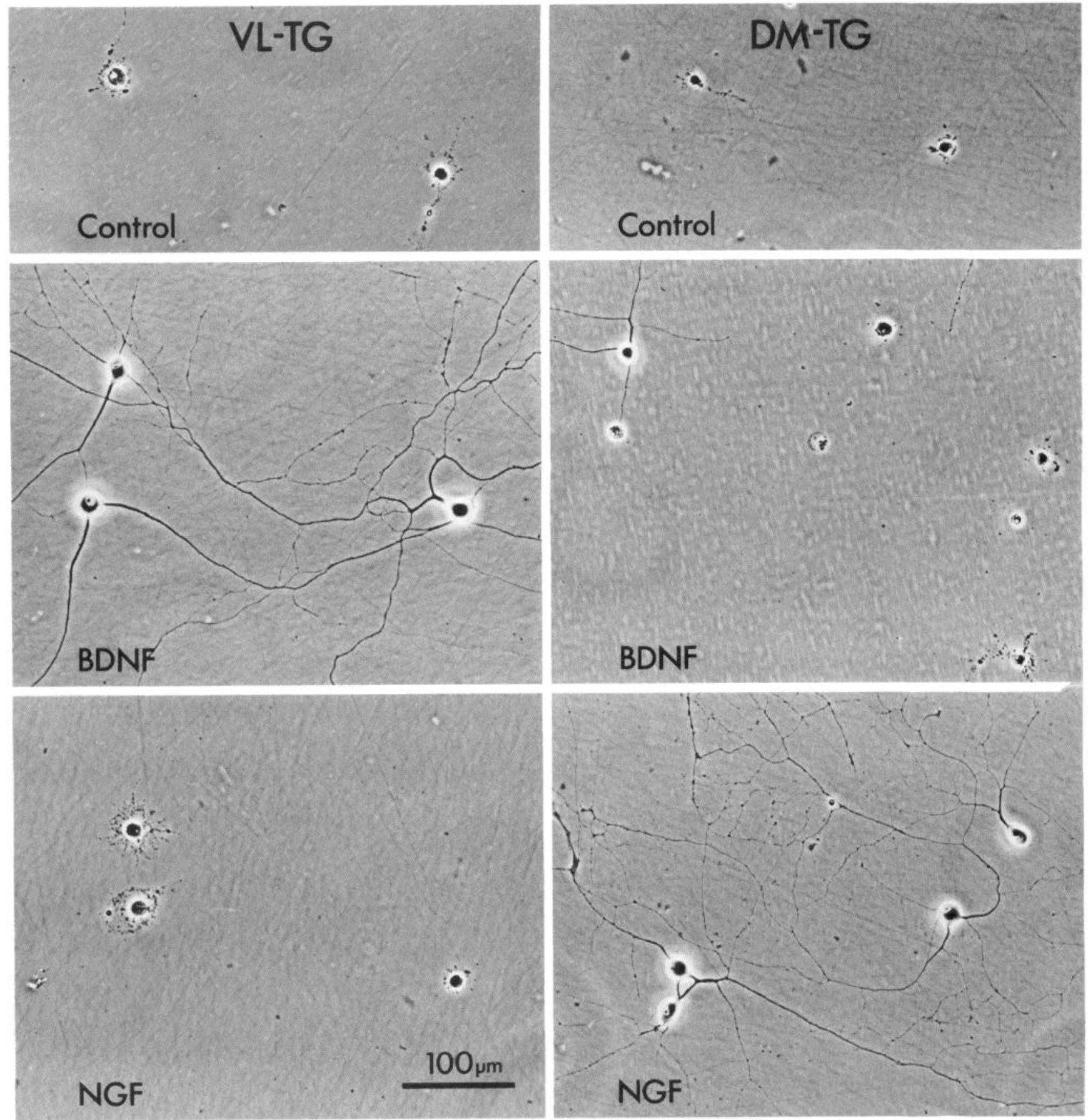

Figure 7. Phase-contrast micrographs of dissociated neuron-enriched cultures of E10 ventrolateral trigeminal neurons $(V L-T G)$ and dorsomedial trigeminal neurons $(D M-T G)$ after $48 \mathrm{hr}$ incubation. Control cultures and cultures supplemented with BDNF $(50 \mathrm{ng} / \mathrm{ml})$ and NGF $(25 \mathrm{ng} / \mathrm{ml})$ are shown.

related to the stage of development of the constituent neurons, that is, neurons generated earlier in development responded to BDNF before later generated neurons. For example, VL-TG and nodose neurons, which are generated between E2 and E5 (D'Amico-Martel, 1982; D'Amico-Martel and Noden, 1980), responded to BDNF earlier in development than DM-TG and jugular neurons, which are generated between E4 and E7. This relationship, however, does not seem to apply to either the geniculate ganglion, which responded late in development but whose neurons are generated at the same time as VL-TG and nodose neurons (D'Amico-Martel, 1982), or to DRG, which responded early but whose neurons are generated at much the same time as DM-TG and jugular neurons (Carr and Simpson, 1978).

On the whole, sensory ganglia appeared to respond to BDNF during much the same stage of development as neural crestderived sensory ganglia respond to NGF. The magnitude of the response to BDNF increased with age to reach a maximum between E10 and E12 (Fig. 2). Likewise, the response of neural crest-derived sensory ganglia to NGF shows a similar increase to reach a maximum between E8 and E11 (Davies and Lindsay, 1985). Furthermore, as has been shown here with DRG, the 
onsets of the response to BDNF and NGF are closely related in development, occurring at E4 with BDNF and at E5 with NGF (Fig. 4). For the majority of sensory neurons, it is not precisely known whether the central and peripheral connections form during the same period of development or at different periods. Several detailed studies of vestibulo-acoustic neurons in the embryonic chick, however, have revealed that the central and peripheral synapses of these neurons form during approximately the same period, that is, during the second week of incubation (Whitehead and Morest, 1978, 1980). Should these features turn out to be shared by other sensory neurons, then our findings suggest that BDNF, like NGF, supports the survival of developing sensory neurons during the stage of target-field innervation.

The location of BDNF in the CNS suggests that this molecule provides trophic support to sensory neurons via their central rather than their peripheral branches. In this context it is interesting to note the recent demonstration by Yip and Johnson (1984) that central rhizotomy of neonatal DRG causes a $50 \%$ reduction in neuronal number, indicating that the survival of developing sensory neurons is indeed dependent on trophic support from the CNS.

Although our findings clearly show that BDNF-responsive neurons are present in all sensory ganglia and in the trigeminal mesencephalic nucleus, the results with dissociated neuron-enriched cultures of trigeminal neurons demonstrate that not all sensory neurons respond to BDNF. Whereas the majority of ventrolateral trigeminal neurons survived and grew in the presence of BDNF, only about $7 \%$ of dorsomedial neurons survived in cultures supplemented with this growth factor (Figs. 6 and 7). The low percentage of survival of DM-TG neurons was not due simply to an unsuitable culture environment, since the majority of these neurons survived and grew under similar conditions in the presence of NGF. This marked difference in the response of DM-TG and VL-TG neurons to BDNF was not apparent in explant cultures (see Fig. 1), which suggests that a small percentage of responsive neurons is enough to create a clear response from explants.

The present finding that NGF and BDNF have reciprocal effects on the survival of DM-TG and VL-TG neurons, together with the finding that NGF-responsive sensory neurons are restricted to neural crest-derived sensory ganglia (Davies and Lindsay, 1985), demonstrate that not all BDNF-responsive sensory neurons respond to NGF. Whether BDNF- and NGFresponsive sensory neurons comprise distinct subsets in neural crest-derived sensory ganglia is unclear. The finding that over $50 \%$ of cultured E6 DRG neurons survive and grow with saturating levels of either BDNF or NGF alone, although substantially more neurons respond in the presence of both factors (Lindsay et al., 1985), suggests that there is some partial overlap between BDNF- and NGF-responsive neurons.

The proposal that sensory neurons depend on the supply of both a factor from the periphery and a different factor from the CNS, bascd on the demonstration that cultured proprioccptive neurons are supported by both BDNF and a neurotrophic factor present in skeletal muscle (Davies et al., 1986), suggests that some sensory neurons (e.g., a proportion of DM-TG neurons) respond to a CNS-derived neurotrophic factor distinct from BDNF. Conversely, other neurons (e.g., VL-TG and nodose ganglion neurons) may respond to peripheral target-derived factors other than NGF. It will be interesting to see in future studies if it is possible to associate BDNF-responsiveness with certain functional types of sensory neurons.

\section{References}

Barde, Y.-A., D. Edgar, and H. Thoenen (1980) Sensory neurons in culture: Changing requirements for survival factors during embryonic development. Proc. Natl. Acad. Sci. USA 77: 1199-1203.
Barde, Y.- $\Lambda .$, D. Edgar, and H. Thoenen (1982) Purification of a new neurotrophic factor from mammalian brain. EMBO J. 1: 549-553.

Berg, D. K. (1982) Cell death in neuronal development. Regulation by trophic factors. In Neuronal Development, N. C. Spitzer, ed., pp. 297-331, Plenum, New York.

Bocchini, V., and P. U. Angeletti (1969) The nerve growth factor: Purification as a 30,000 molecular-weight protein. Proc. Natl. Acad. Sci. USA 64: 787-794.

Carr, V. M., and S. B. Simpson (1978) Proliferative and degenerative events in the early development of chick dorsal root ganglia: Normal development. J. Comp. Neurol. 182: 727-740.

Collins, F. (1978) Axon initiation by ciliary neurons in culture. Dev. Biol. 65: 50-57.

Cowan, W. M., J. W. Fawcett, D. D. M. O'Leary, and B. B. Stanfield (1984) Regressive events in neurogenesis. Science 225: 1258-1265. D'Amico-Martel, A. (1982) Temporal patterns of neurogenesis in avian cranial sensory and autonomic ganglia. Am. J. Anat. 163: 351372.

D'Amico-Martel, A., and D. M. Noden (1980) An autoradiographic analysis of the development of the chick trigeminal ganglion. J. Embryol. Exp. Morphol. 55: 167-182.

Davies, A. M. (in press) The survival and growth of embryonic proprioceptive neurons is promoted by a factor present in skeletal muscle. Dev. Biol.

Davies, A. M., and R. M. Lindsay (1984) Neural crest-derived spinal and cranial sensory neurons are equally sensitive to NGF but differ in their response to tissue extracts. Dev. Brain Res. 14: 121-127.

Davies, A. M., and R. M. Lindsay (1985) The cranial sensory ganglia in culture: Differences in the response of placode-derived and neural crest-derived neurons to nerve growth factor. Dev. Biol. 111: 62-72.

Davies, A. M., and A. G. S. Lumsden (1983) Influence of nerve growth factor on developing dorso-medial and ventro-lateral neurons of chick and mouse trigeminal ganglia. Int. J. Dev. Neurosci. 1: 171-177.

Davies, A. M., and A. G. S. Lumsden (1984) Relation of target encounter and neuronal death to nerve growth factor responsiveness in the developing mouse trigeminal ganglion. J. Comp. Neurol. 223: 124-137.

Davies, A. M., H. Thoenen, and Y.-A. Barde (1986) Different factors from the central nervous system and periphery regulate the survival of sensory neurons. Nature 319: 497-499.

Edgar, D., R. Timpl, and H. Thoenen (1984) The heparin-binding domain of laminin is responsible for its effects on neurite outgrowth and neuronal survival. EMBO J. 3: 1463-1468.

Korsching, S., and H. Thoenen (1983) Nerve growth factor in sympathetic ganglia and corresponding target organs of the rat: Correlation with density of sympathetic innervation. Proc. Natl. Acad. Sci. USA 80: 3513-3516.

Lindsay, R. M., and H. Rohrer (1985) Placodal sensory neurons in culture. Nodose ganglion neurons are unresponsive to NGF, lack NGF receptors but are supported by a liver-derived neurotrophic factor. Dev. Biol. 112: 30-48.

Lindsay, R. M., and J. Tarbit (1979) Developmentally regulated induction of neurite outgrowth from immature chick sensory neurons (DRG) by homogenates of avian or mammalian heart, liver and brain. Neurosci. Lett. 12: 195-200.

Lindsay, R. M., H. Thoenen, and Y.-A. Barde (1985) Placode and neural crest-derived sensory neurons are responsive at early developmental stages to brain-derived neurotrophic factor (BDNF). Dev. Biol. 112: 319-328.

Lumsden, A. G. S., and A. M. Davies (1983) Earliest sensory nerves are guided to peripheral targets by attractants other than nerve growth factor. Nature 306: 786-788.

Oppenheim, K. W. (1981) Neuronal death and some related regressive phenomena during neurogenesis: A selective historical review and progress report. In Studies in Developmental Biology, W. M. Cowan, ed., pp. 74-133, Oxford U. P., Oxford.

Suda, K., Y.-A. Barde, and H. Thoenen (1978) Nerve growth factor in mouse and rat serum: Correlation between bioassay and radioimmunoassay determinations. Proc. Natl. Acad. Sci. USA 75: 40424046.

Thoenen, II., and Y.-A. Barde (1980) Physiology of nerve growth factor. Physiol, Rev. 60: 1284-1335.

Whitehead, M. C., and D. K. Morest (1978) Morphogenesis of synaptic endings of cochlear fibres in the chick basilar papilla. Soc. Neurosci. Abstr. 4: 130. 
Whitehead, M. C., and D. K. Morest (1980) Nerve-target cell interactions during development of the avian inner ear. Soc. Neurosci. Abstr. 6: 820

Yip, H. K., and E. M. Johnson (1984) Developing dorsal root ganglion neurons require trophic support from their central processes: Evidence for a role of retrogradely transported nerve growth factor from the central nervous system to the periphery. Proc. Natl. Acad. Sci. USA 81: 6245-6249. 